\title{
OPEN A bagging dynamic deep learning network for diagnosing COVID-19
}

\author{
Zhijun Zhang ${ }^{1,2,3,4,5 \bowtie}$, Bozhao Chen ${ }^{1}$, Jiansheng Sun ${ }^{1} \&$ Yamei Luo $^{1}$
}

\begin{abstract}
COVID-19 is a serious ongoing worldwide pandemic. Using X-ray chest radiography images for automatically diagnosing COVID-19 is an effective and convenient means of providing diagnostic assistance to clinicians in practice. This paper proposes a bagging dynamic deep learning network (B-DDLN) for diagnosing COVID-19 by intelligently recognizing its symptoms in X-ray chest radiography images. After a series of preprocessing steps for images, we pre-train convolution blocks as a feature extractor. For the extracted features, a bagging dynamic learning network classifier is trained based on neural dynamic learning algorithm and bagging algorithm. B-DDLN connects the feature extractor and bagging classifier in series. Experimental results verify that the proposed B-DDLN achieves $98.8889 \%$ testing accuracy, which shows the best diagnosis performance among the existing state-of-the-art methods on the open image set. It also provides evidence for further detection and treatment.
\end{abstract}

COVID-19 has been rapidly and widely spreading as an ongoing pandemic throughout the world since the end of 2019 and has been responsible for a high number of fatalities ${ }^{1-3}$. According to statistics on the Worldometers. info website, over 198 million people have been diagnosed with COVID-19 and about 4.22 million deaths have occurred up to the end of July, 2021. Most detection methods for COVID-19, including nucleic acid amplification testing, rely on pathogen testing ${ }^{4}$. However, pathogen testing has some limitations. First of all, it requires testing kits that have limited availability in the supply chain ${ }^{5}$. Secondly, it is time-consuming because the detection steps are tedious and have high technical requirements ${ }^{6,7}$.

Given the limitations of nucleic acid amplification testing, the recognition of COVID-19 indications in X-ray chest radiography images can be performed to identify COVID-19 patients, which makes the diagnosis of the disease and its severity more intuitive ${ }^{8,9}$. This is a vital verification method with simple operation. The equipments required for X-ray chest radiography are lightweight and transportable ${ }^{10}$. The entire recognition process takes much less time (about $15 \mathrm{~s}$ per patient ${ }^{10}$ ) and manual operations to give the final visualized results, thus providing considerable assistance in a convenient manner to clinicians in practice ${ }^{11}$. Especially in China, many cases can be identified as suspected COVID-19 infections if the characteristic manifestations are observed in X-ray scans s $^{11,12}$. In the field of intelligent medical diagnosis, deep learning models have been widely developed and applied in recent years. They give their excellent abilities of feature extraction and classification ${ }^{13}$. An increasing number of researchers have applied deep learning and computer vision technologies to diagnose COVID-19 by recognizing medical images ${ }^{14}$. For example, Zhang et al. proposed a confidence-aware anomaly detection model that consists of a shared feature extractor, an anomaly detection module, and a confidence prediction module. The model achieved an AUC of 0.8361 and a sensitivity of $0.7170^{15}$. To further improve the diagnostic effect, Majeed et al. designed a convolutional neural network (CNN) model for COVID-19 detection from X-ray chest radiography images that obtained 0.9315 sensitivity and 0.9786 specificity ${ }^{16}$. Singh et al. proposed a deep convolutional neural network, whose hyper-parameters can be tuned by using multi-objective adaptive differential evolution. Their model achieved $94.48 \%$ testing accuracy ${ }^{17}$. To accelerate diagnosis, Brunese et al. considered a deep learning network based on a VGG-16 model by exploiting transfer learning with an average time of approximately $2.5 \mathrm{~s}$ and $97.00 \%$ average accuracy ${ }^{18}$. On the face of it, the accuracy and related performance indicate a great diagnosis level with a low misdiagnosis rate. However, without shortcut connections in these deep learning models, the vanishing-gradient problem may occur during the training process.

To effectively solve the vanishing-gradient problem, He et al. proposed a residual convolutional neural network (ResNet) in 2015 by adding shortcut connections and enhancing training efficiency efficiency ${ }^{19-22}$. Inspired

\footnotetext{
${ }^{1}$ School of Automation Science and Engineering, South China University of Technology, Guangzhou 510640, China. ${ }^{2}$ Guangdong Artificial Intelligence and Digital Economy Laboratory (Pazhou Lab), Guangzhou 510335, China. ${ }^{3}$ School of Automation Science and Engineering, East China Jiaotong University, Nanchang 330052, China. ${ }^{4}$ Shaanxi Provincial Key Laboratory of Industrial Automation, School of Mechanical Engineering, Shaanxi University of Technology, Hanzhong 723001, China. ${ }^{5}$ School of Information Technology and Management, Hunan University of Finance and Economics, Changsha 410205, China. ${ }^{\bowtie}$ email: auzjzhang@scut.edu.cn
} 
by the method in reference ${ }^{20}$, Luz et al. applied ResNet50 to COVID-19 detection in X-ray images, which achieved 93.9\% overall accuracy, $96.8 \%$ sensitivity, and $100 \%$ positive prediction ${ }^{23}$. Based on the fine-tuned ResNet model, Farooq et al. developed a deep learning framework called COVID-ResNet to screen COVID-19 from radiographs that achieved $96.23 \%$ accuracy and $100 \%$ sensitivity with only 41 epochs ${ }^{24}$. For further developments of the ResNet, densely connected convolutional networks (DenseNet) was proposed in 2017 by designing dense blocks, which alleviated the vanishing-gradient problem, and strengthened feature propagation, thus encouraging feature reuse and substantially reducing the number of parameters effectively ${ }^{25}$. Based on DenseNet, Wang et al. designed and proposed COVID-Net architecture for COVID-19 diagnosis that achieved 93.33\% accuracy by recognizing X-ray images of normal, COVID-19, and ordinary pneumonia cases ${ }^{26}$. To improve the models' initial performance, Narayan Das et al. combined deep transfer learning with the Xception model for COVID19 diagnosis, which could achieve $97.41 \%$ testing accuracy ${ }^{27}$. However, the generalization performance of fully connected layer at the end of ResNet-based models may not be strong enough to discriminate and classify deep convolutional features ${ }^{28}$.

For improving the generalization performance of the classifier, support vector machine (SVM) is an appropriate choice to classify samples precisely by searching for the optimal decision boundary. Researchers have combined CNN and SVM for image recognition, where CNN is applied for feature extraction and SVM classifies deep extracted features. For COVID-19 diagnosis, Sethy et al. used SVM to classify deep image features extracted from the fully connected layer of ResNet50, which achieved $95.38 \%$ diagnosis accuracy ${ }^{29}$. To enhance the classification efficiency, Novitasari et al. removed the fully connected layers of the pre-trained ResNet 18 as a feature extractor. For the image features extracted from the global average pooling layer, they utilized principal component analysis and relief methods in feature selection and applied SVM as a classifier, which achieved $96.15 \%$ diagnosis accuracy ${ }^{30}$. However, for the nonlinear distribution of samples, we should find an appropriate kernel function to map these samples into linear separated space, which requires too many computational resources and too much model training time.

To accelerate training models with less computational overhead, neural dynamic learning algorithm (NDLA) has been proposed under the restrictions of high real-time requirement and limited hardware resources. With the characteristics of error exponential convergence and parallel computing, NDLA has been used to train some neural networks. For example, in order to solve time-varying convex quadratic programming problems constrained by linear equality and obtain online solutions to the time-varying Sylvester equation, a varyingparameter convergent-differential neural network (VP-CDNN) was proposed by using an exponential-type time varying design function ${ }^{31,32}$. Related theoretical analysis has proved that using VP-CDNN can make the residual error over the continuous time converge to zero super-exponentially. Based on the proposed VP-CDNN, various practical problems in time-varying over-determined system ${ }^{33}$, time-varying complex Sylvester equation ${ }^{34}$, disturbed time-varying inversion systems ${ }^{35}$, robot tracking ${ }^{36,37}$, and unmanned aerial vehicle controller ${ }^{38}$ have been effectively solved. Zhang et al. designed adaptive multi-layer neural dynamics-based controllers of multirotor unmanned aerial vehicles that can adapt to various complex transportation needs ${ }^{39}$. In addition, to solve the time-varying quadratic programming problem and robot tracking problem, a power-type varying-parameter recurrent neural network was proposed that is different from VP-CDNN ${ }^{40,41}$. To solve the non-repetitive motion problem of redundant robot manipulators, Zhang et al. proposed an adaptive fuzzy recurrent neural network that can avoid the saturation of time-varying design functions ${ }^{42}$. For application in machine learning and pattern recognition, a voting convergent difference neural network was proposed for diagnosing the presence of breast cancer and breast tumor types that achieved $100 \%$ diagnosis accuracy ${ }^{43}$. Thus, NDLA has faster convergence rate and higher accuracy ${ }^{33}$, and a network trained by an NDLA is more suitable for solving problems that have challenging real-time requirements with limited hardware resources. However, to the best of the authors' knowledge, NDLA-based networks have not yet been applied in the field of deep learning and image classification.

For a faster and more accurate COVID-19 diagnosis, this paper proposes a bagging dynamic deep learning network (B-DDLN) that consists of two modules: a feature extractor and a bagging classifier. In the proposed B-DDLN, five pre-trained convolution blocks are used as a feature extractor for X-ray chest radiography images and a bagging classifier based on NDLA and bagging algorithm is applied to classify these image features. The structure of the bagging classifier is lightweight and its cost function is more easily designed in comparison to most classifiers. The proposed B-DDLN can rapidly make the training and testing errors converge and enhance the diagnosis precision. Owing to the utilization of a bagging classifier, B-DDLN can solve class-imbalance problems by random under-sampling and further enhance the diagnosis results' accuracy and reliability.

\section{Bagging dynamic deep learning network}

In this section, the proposed bagging dynamic deep learning network (B-DDLN) is designed and analyzed detailedly in four stages. First of all, the construction of the B-DDLN is proposed. Secondly, the principle of dynamic learning network are presented for designing a classifier. Thirdly, neural dynamic learning algorithm (NDLA) of a dynamic learning network is derived in detail. Fourthly, based on bootstrap aggregation and the voting decision method among several dynamic learning networks trained by NDLA with various mapping functions, a bagging dynamic learning network classifier is proposed to enhance the generalization performance of a dynamic learning network.

Construction of B-DDLN diagnosis model. For the application of medical image classification, a convolutional neural network is very effective because of its convolution blocks for extracting differentiated image features ${ }^{19,20}$. However, the generalization performance of fully connected layers in CNNs may not be strong enough to discriminate and classify deep convolutional features ${ }^{28}$. For diagnosing COVID-19, the accuracy and precision cannot be stabilized at a satisfactory level when only a CNN is used. To enhance the generalization 


\section{B-DDLN Diagnosis Model}

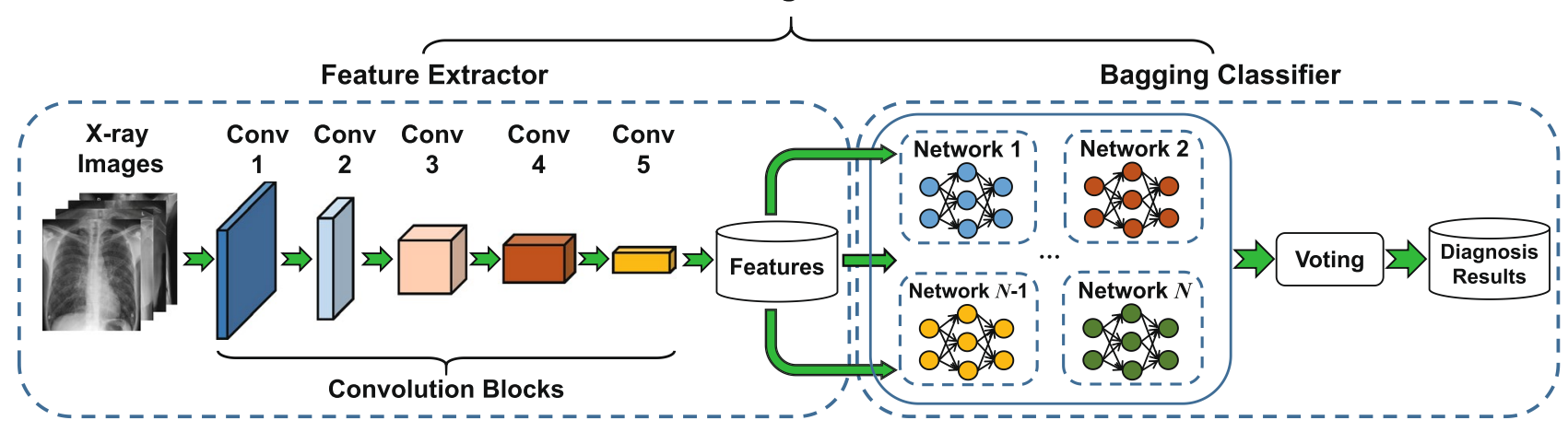

Figure 1. Construction of the proposed bagging dynamic deep learning network (B-DDLN) diagnosis model. The convolution blocks consist of convolutional layers, pooling layers, batch normalization, ReLU layers, and shortcut connections. In the feature extractor, five convolution blocks are designed and pre-trained for extracting the features of X-ray chest radiography images, and a bagging dynamic learning network classifier that consists of $N$ unit dynamic learning networks is responsible for recognizing these features to generate the final diagnosis results.

performance of diagnosis model, B-DDLN is proposed in this paper, which consists of two modules: a feature extractor and a bagging classifier. In the feature extractor, the convolution blocks consist of convolutional layers, pooling layers, batch normalization, ReLU layers and shortcut connections. They are designed and pre-trained as a whole feature extractor for X-ray chest radiography images, and bagging dynamic learning network classifier based on NDLA and bagging algorithm is applied to classify these image features and generate diagnostic results.

Figure 1 shows how the proposed B-DDLN is constructed. We use images from training set to pre-train five designed convolution blocks as a feature extractor. Suppose a three-channel image tensor $\boldsymbol{g}_{s} \in \mathbb{R}^{224 \times 224 \times 3}$ is input into this feature extractor, its corresponding feature vector $h\left(g_{s}\right) \in \mathbb{R}^{1 \times 512}$ can be obtained. After gathering all the features of the training images from the feature extractor, we use these features to train the bagging dynamic learning network classifier and evaluate the complete proposed B-DDLN. The bagging dynamic learning network classifier consists of $N$ dynamic learning networks, where $N$ denotes the number of dynamic learning networks. These $N$ dynamic learning networks are trained by using $N$ subsets randomly collected from all the extracted training feature samples and NDLA with $N$ different types of mapping functions.

As the proposed B-DDLN diagnosis model combines convolution blocks as a feature extractor and bagging dynamic learning network classifier, the constructed principle is termed B-DDLN constructed algorithm. Algorithm 1 illustrates the steps in detail.

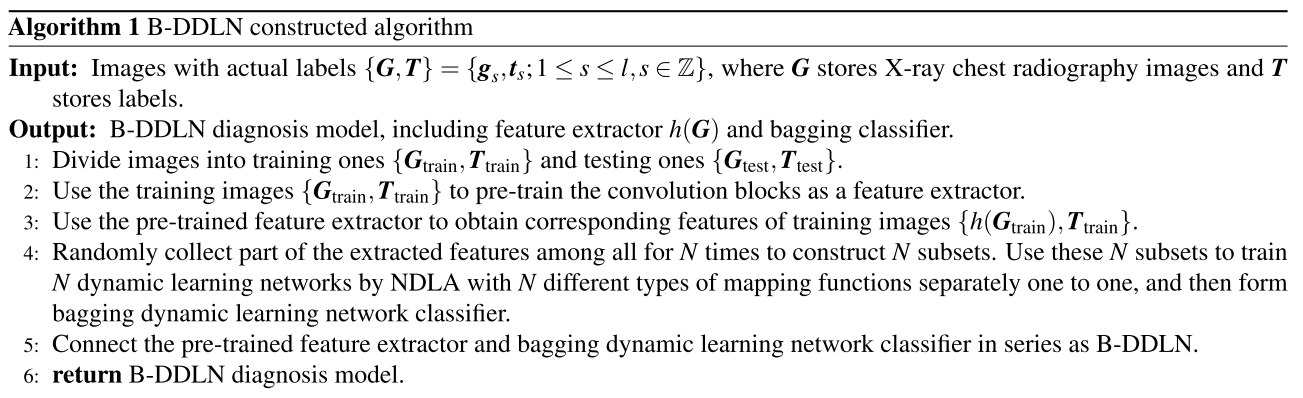

Topology and principle of dynamic learning network. A three-layer dynamic learning network is designed and proposed in the bagging classifier module of the proposed B-DDLN. Some variables are explained as below:

$l$ : Number of input samples.

$m$ : Feature dimensions.

$n$ : Number of hidden neurons.

$q$ : Number of classes for samples.

$\boldsymbol{X}$ : An $l \times m$ input matrix storing $l$ samples with $m$ dimensions. $x_{s i}(1 \leq s \leq l, 1 \leq i \leq m ; s, i \in \mathbb{Z}) \in \boldsymbol{X}$ represents the value of the $i$ th dimension for the $s$ th sample.

$V$ : An $m \times n$ matrix storing weights connecting input and hidden layers. $v_{i j}(1 \leq i \leq m, 1 \leq j \leq n ; i, j \in \mathbb{Z}) \in V$ represents the weight component connecting the $i$ th input neuron with the $j$ th hidden neuron.

I: An $l \times n$ matrix input into the hidden layer. 
Q: An $l \times n$ hidden output matrix correspond to $\boldsymbol{I}$.

$W$ : An $n \times q$ matrix storing weights connecting hidden and output layers. $w_{j r}(1 \leq j \leq n, 1 \leq r \leq q$; $j, r \in \mathbb{Z}) \in W$ represents the weight component connecting the $j$ th hidden neuron with the $r$ th output neuron. $\boldsymbol{Y}:$ An $l \times q$ predicted diagnosis output matrix.

$f_{j}(\cdot)(1 \leq j \leq n ; j \in \mathbb{Z})$ : Activation functions in the $j$ th hidden neurons.

$g(\cdot)$ : Activation function in the output neurons.

$\bar{Y}, L: l \times q$ label matrices, where $\overline{\boldsymbol{Y}}$ is encoded as $-1-1$ format and $\boldsymbol{L}$ is encoded by one-hot vectors.

$P$ : An $l \times q$ class possibility matrix calculated by softmax formula.

$\varepsilon$ : Training error of dynamic learning network calculated by cross entropy formula.

The feed-forward output of the dynamic learning network is formulated as

$$
\boldsymbol{Y}=g(\boldsymbol{Q W})=g(\boldsymbol{F}(\boldsymbol{I}) \boldsymbol{W}) \in \mathbb{R}^{l \times q}
$$

where

$$
\begin{gathered}
\boldsymbol{X}=\left[\begin{array}{lll}
x_{11} & \cdots & x_{1 m} \\
\vdots & \ddots & \vdots \\
x_{l 1} & \cdots & x_{l m}
\end{array}\right] \in \mathbb{R}^{l \times m} \\
\boldsymbol{Q}=\boldsymbol{F}(\boldsymbol{I})=\boldsymbol{F}(\boldsymbol{X} \boldsymbol{V}) \in \mathbb{R}^{l \times n} \\
\boldsymbol{F}(\boldsymbol{I})=\left[f_{1}\left(\boldsymbol{i}_{1}\right) ; f_{2}\left(\boldsymbol{i}_{2}\right) ; f_{3}\left(\boldsymbol{i}_{3}\right) ; \ldots ; f_{j}\left(\boldsymbol{i}_{j}\right) ; \ldots ; f_{n}\left(\boldsymbol{i}_{n}\right)\right] \\
\boldsymbol{W}=\left[\begin{array}{lll}
w_{11} & \cdots & w_{1 q} \\
\vdots & \ddots & \vdots \\
w_{n 1} & \cdots & w_{n q}
\end{array}\right] \in \mathbb{R}^{n \times q} .
\end{gathered}
$$

In Eq. (4), $\boldsymbol{i}_{j} \in \mathbb{R}^{l \times 1}$ denotes the $j$ th column vector of $\boldsymbol{I} \in \mathbb{R}^{l \times n}$. For activation functions in the hidden and output neurons, two cases are listed as examples, i.e.,

Case 1: All the activation functions are set as softsign function. The expression is

$$
f_{j}(z)=g(z)=\frac{z}{1+|z|}(1 \leq j \leq n ; j \in \mathbb{Z}) .
$$

Case 2: Activation functions of the output neurons are still set as softsign function, while those of the hidden neurons are power-softsign function. The expression of power-softsign function is

$$
f_{j}(z)=\left(\frac{z}{1+|z|}\right)^{j-1}(1 \leq j \leq n ; j \in \mathbb{Z})
$$

For the sth sample $\boldsymbol{x}_{s}=\left[x_{s 1}, \ldots, x_{s m}\right] \in \mathbb{R}^{1 \times m}(1 \leq s \leq l ; s \in \mathbb{Z})$ from $\boldsymbol{X}$, the output through dynamic learning network $\boldsymbol{y}_{s}=\left[y_{s 1}, \ldots, y_{s q}\right] \in \mathbb{R}^{1 \times q}$ is calculated using Eq. (1), and the corresponding class probabilities vector $\boldsymbol{P}_{s}$ is obtained using the softmax formula

$$
\boldsymbol{P}_{s}=\left[P_{s 1}, \ldots, P_{s q}\right]=\left[\frac{\exp \left(y_{s 1}\right)}{\sum_{r=1}^{q} \exp \left(y_{s r}\right)}, \ldots, \frac{\exp \left(y_{s q}\right)}{\sum_{r=1}^{q} \exp \left(y_{s r}\right)}\right] \in \mathbb{R}^{1 \times q}
$$

where $P_{s 1}, \ldots, P_{s q} \in[0,1], \sum_{r=1}^{q} P_{s r}=1$. If $P_{s r}=\max \left\{P_{s 1}, \ldots, P_{s q}\right\}(1 \leq r \leq q ; r \in \mathbb{Z}), \boldsymbol{x}_{s}$ is predicted as belonging to the $r$ th class according to Bayesian decision principle based on the minimum classification error probability $^{44}$. Similarly, the class probability matrix $\boldsymbol{P}$ for $l$ input samples is calculated below.

$$
\boldsymbol{P}=\left[\begin{array}{lll}
P_{11} & \cdots & P_{1 q} \\
\vdots & \ddots & \vdots \\
P_{l 1} & \cdots & P_{l q}
\end{array}\right]=\left[\begin{array}{lll}
\frac{\exp \left(y_{11}\right)}{\sum_{r=1}^{q} \exp \left(y_{1 r}\right)} & \cdots & \frac{\exp \left(y_{1 q}\right)}{\sum_{r=1}^{q} \exp \left(y_{1 r}\right)} \\
\vdots & \ddots & \vdots \\
\frac{\exp \left(y_{l 1}\right)}{\sum_{r=1}^{q} \exp \left(y_{l r}\right)} & \cdots & \frac{\exp \left(y_{l q}\right)}{\sum_{r=1}^{q} \exp \left(y_{l r}\right)}
\end{array}\right] \in \mathbb{R}^{l \times q}
$$

Learning algorithm of dynamic learning network. Neural dynamic learning algorithm (NDLA) has been favored in recent years owing to more rapid convergence and higher precision ${ }^{40,45}$. Its design formula is expressed as

$$
\dot{e}(t)=-\lambda \Phi(e(t))
$$




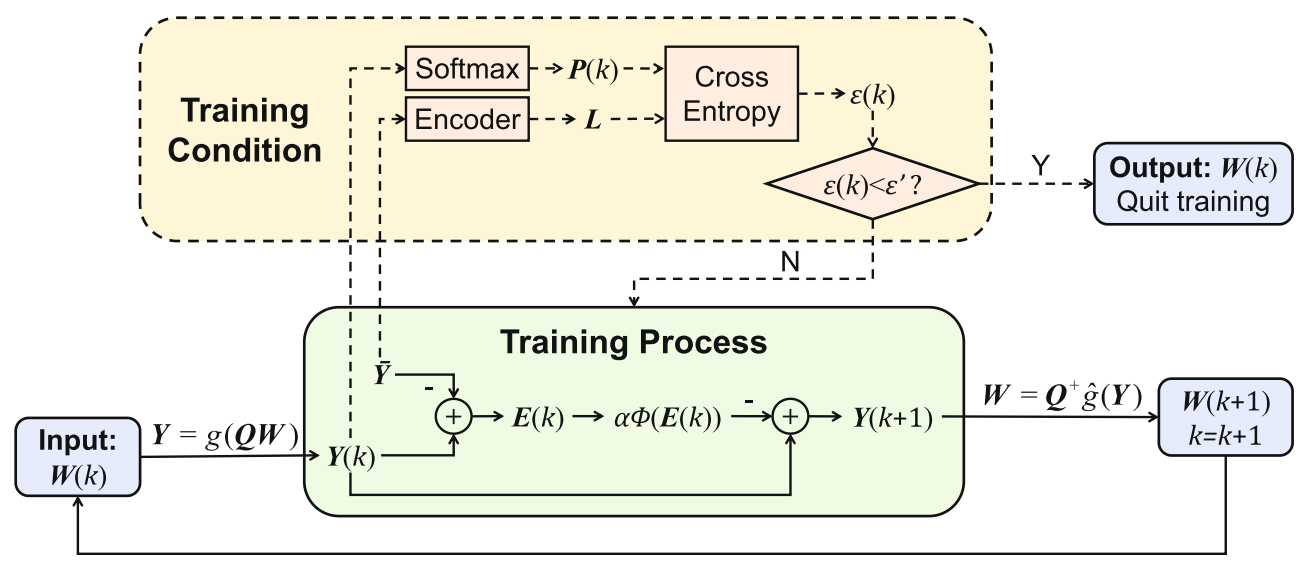

Figure 2. Diagram of neural dynamic learning algorithm (NDLA). If training error $\varepsilon(k)$ calculated by the class possibility matrix $\boldsymbol{P}(k)$ and the label matrix $\boldsymbol{L}$ satisfies $\varepsilon(k) \geq \varepsilon^{\prime}$, where $\varepsilon^{\prime}$ denotes a threshold, the weight matrix connecting between hidden and output layers $W(k)$ will be updated as $W(k+1)$ at the $(k+1)$ th training round under the situation of known NDLA design coefficient $\alpha$, mapping function $\Phi(\cdot)$, hidden output matrix $\boldsymbol{Q}$, the diagnosis deviation matrix $\boldsymbol{E}$, predicted diagnosis output matrix $\boldsymbol{Y}$, and the label matrix $\overline{\boldsymbol{Y}}$.

In Eq. (10), $t \in \mathbb{R}$ denotes continuous time. $e(t) \in \mathbb{R}$ denotes deviation between prediction output and expectation over $t . \dot{e}(t)$ denotes the derivative of the deviation $e(t)$ with respect to $t . \lambda>0, \lambda \in \mathbb{R}$ denotes NDLA parameter. $\Phi(\cdot)$ denotes mapping function that is a monotonically increasing and odd function ${ }^{38,40,43,45}$. NDLA is conducted in digital computer. Such serial time expression (i.e., Eq. (10)) is transformed into a discrete ones combined with Euler discrete formula ${ }^{46}$, which is shown below.

$$
\frac{e(k+1)-e(k)}{\tilde{h}}=-\lambda \Phi(e(k))
$$

where $\tilde{h}>0, \tilde{h} \in \mathbb{R}$ denotes the discrete step, and $k>0, k \in \mathbb{Z}$ denotes discrete time and the $k$ th training round. Equation (11) is further transformed as

$$
e(k+1)=e(k)-\alpha \Phi(e(k)) \in \mathbb{R}
$$

where $\alpha=\tilde{h} \lambda>0, \alpha \in \mathbb{R}$ denotes NDLA design coefficient. Thus, Eq. (12) is a discrete NDLA design formula.

Theorem 1 A deviation based learning rule is formulated as a discrete NDLA design formula, i.e., $e(k+1)-e(k)=-\alpha \Phi(e(k))$, where $\alpha>0$ is a proper value within a range. If the mapping function $\Phi(\cdot)$ in NDLA is a monotonically increasing and odd function, then the deviation between prediction output and expectation e $(k)$ shows absolute convergence to zero along with discrete time $k$ increasing.

Proof The discrete NDLA design formula is expressed as

$$
e(k+1)-e(k)=-\alpha \Phi(e(k)) .
$$

If the mapping function $\Phi(\cdot)$ is a monotonically increasing and odd function, $\Phi(e(k))>0$ is obtained when the deviation $e(k)$ satisfies $e(k)>0$. Because NDLA coefficient satisfies $\alpha>0,-\alpha \Phi(e(k))<0$ is then deduced, which leads to $e(k+1)<e(k)$ according to Eq. (13). Conversely, when the deviation $e(k)<0,-\alpha \Phi(e(k))>0$ is obtained, and $e(k+1)>e(k)$ can be deduced further. Thus, $|e(k+1)|<|e(k)|$ is deduced. With the discrete time $k$ increasing, the absolute value of the deviation will absolutely converge to zero, which is expressed as $\lim _{k \rightarrow+\infty}|e(k)|=0$. The proof is completed.

For the mapping function, the linear, tanh and sinh types are applied as examples in this paper, i.e.,

$$
\begin{gathered}
\Phi_{1}(z)=z \\
\Phi_{2}(z)=\tanh (z) \\
\Phi_{3}(z)=\sinh (z) .
\end{gathered}
$$

The discrete NDLA design formula in this paper is used to train dynamic learning network, in which the weight matrix $\boldsymbol{W}$ is iterated. As shown in Fig. 2, when the $k$ th training round is finished, the diagnosis deviation matrix $\boldsymbol{E}(k)$ is calculated by 


$$
\boldsymbol{E}(k)=\boldsymbol{Y}(k)-\overline{\boldsymbol{Y}} \in \mathbb{R}^{l \times q}
$$

where $\boldsymbol{Y}(k)$ denotes output through dynamic learning network and $\bar{Y}$ denotes labels of samples in $-1-1$ format. Before the next round, the training error $\varepsilon(k)$ is calculated by cross entropy formula at first, i.e.,

$$
\varepsilon(k)=\frac{1}{l} \sum_{s=1}^{l} \sum_{r=1}^{q}\left(-L_{s r} \log \left(P_{s r}\right)\right) \in \mathbb{R}
$$

where $L_{s r} \in \boldsymbol{L}$ and $P_{s r} \in \boldsymbol{P}(k)$. The class probability matrix $\boldsymbol{P}(k)$ is calculated according to Eq. (9). Suppose the threshold of training error is $\varepsilon^{\prime} \in \mathbb{R}$. If $\varepsilon(k)<\varepsilon^{\prime}$, stop and quit this training process. Otherwise, continue updating $\boldsymbol{W}$.

For training details of dynamic learning network in the $(k+1)$ th training round, the diagnosis deviation matrix $\boldsymbol{E}(k+1)$ is obtained by the following equation, i.e.,

$$
\boldsymbol{E}(k+1)=\boldsymbol{E}(k)-\alpha \Phi(\boldsymbol{E}(k)) \in \mathbb{R}^{l \times q}
$$

where

$$
\boldsymbol{E}(k+1)=\boldsymbol{Y}(k+1)-\overline{\boldsymbol{Y}} \in \mathbb{R}^{l \times q} .
$$

When $\Phi(\cdot)$ is a monotonically increasing and odd function, $\boldsymbol{E}(k)$ can rapidly converge to $\mathbf{0}$ along with $k$ increasing according to Theorem 1, which indicates that $\lim _{k \rightarrow+\infty} \boldsymbol{Y}(k)=\overline{\boldsymbol{Y}}$ according to Eq. (17). Through softmax formula (i.e., Eq. (9)) and encoder, $\lim _{k \rightarrow+\infty} \boldsymbol{P}(k)=\overline{\boldsymbol{P}}$ is obtained, where $\overline{\boldsymbol{P}}$ denotes a constant targeted class probability matrix. Thus, the training error $\varepsilon(k)$ satisfies $\lim _{k \rightarrow+\infty} \varepsilon(k)=\tilde{\varepsilon}$ according to Eq. (18), which indicates that $\varepsilon(k)$ theoretically converges to a targeted constant $\tilde{\varepsilon}$ along with $k$ increasing.

Substituting Eqs. (17) and (20) to Eq. (19), we can obtain discrete neural dynamic equation

$$
\boldsymbol{Y}(k+1)=\boldsymbol{Y}(k)-\alpha \Phi(\boldsymbol{E}(k)) \in \mathbb{R}^{l \times q}
$$

where

$$
\begin{gathered}
\boldsymbol{Y}(k)=g(\boldsymbol{Q W}(k)) \in \mathbb{R}^{l \times q} \\
\boldsymbol{Y}(k+1)=g(\boldsymbol{Q} \boldsymbol{W}(k+1)) \in \mathbb{R}^{l \times q} .
\end{gathered}
$$

Therefore, $\boldsymbol{W}(k+1)$ can be figured out under the situation of known $\boldsymbol{Q}$ and $\boldsymbol{W}(k)$. Substituting Eqs. (22) and (23) to Eq. (21), we can obtain

$$
g(\boldsymbol{Q W}(k+1))=g(\boldsymbol{Q W}(k))-\alpha \Phi(\boldsymbol{E}(k)) \in \mathbb{R}^{l \times q} .
$$

$W(k+1)$ is figured out by solving Eq. (24) as

$$
\boldsymbol{W}(k+1)=\boldsymbol{Q}^{+} g^{-1}(g(\boldsymbol{Q W}(k))-\alpha \Phi(\boldsymbol{E}(k))) \in \mathbb{R}^{n \times q} .
$$

Equation (25) shows the iterated relation between $\boldsymbol{W}(k+1)$ and $\boldsymbol{W}(k)$. A training round is thus finished. Without loss of generality, the softsign function is applied as an example of $g(\cdot)$. However, the inverse function of softsign function can not be found. Under this circumstance, an approximate expression $\hat{g}(\cdot)$ is used instead, i.e.,

$$
g^{-1}(z) \approx \hat{g}(z)=\frac{z}{1-|z|} .
$$

Bagging dynamic learning network classifier. To further enhance the generalization of the dynamic learning network, bootstrap aggregating (bagging) algorithm is utilized to construct a more robust bagging dynamic learning network classifier, as illustrated in Fig. 1. In bagging algorithm, some of the samples are randomly collected from all the training features as a subset, which is used to train a dynamic learning network. This process is repeated for several times, and several trained dynamic learning networks are obtained. In combination strategy, these trained dynamic learning networks start by predicting testing samples. Then with regard to these predicted results, the final belonging class is determined based on the principle of plurality voting, i.e., the majority rule ${ }^{43,47}$. Specifically, we initialize a zero vector $\boldsymbol{a}_{t}=\left[a_{t 1}, \ldots, a_{t q}\right] \in \mathbb{R}^{1 \times q}$ for the vote statistic. If the $\hat{k}$ th dynamic learning network model determines that the testing sample $\boldsymbol{x}_{t}=\left[x_{t 1}, \ldots, x_{t m}\right] \in \mathbb{R}^{1 \times m}$ belongs to the $\hat{r}$ th class $(\hat{k} \in[1, N], \hat{r} \in[1, q] ; \hat{k}, \hat{r} \in \mathbb{Z}), a_{t} \hat{r}$ will be increased by one. Based on the majority rule, we determine that the index corresponding to the maximum number of votes among all the elements in $\boldsymbol{a}_{t}$ is the final predicted class after vote statistic.

Structure and principle of bagging dynamic learning network classifier are shown in Fig. 3, where dynamic learning networks trained by NDLA using various mapping functions $\Phi(\cdot)$ are simultaneously trained by using various subsets drawn randomly from shuffled training features. Suppose there are two kinds of diagnosis results (positive and negative) and $N$ dynamic learning networks. When $\boldsymbol{x}_{t}$ is entered into this proposed bagging dynamic learning network, according to the majority rule, these dynamic learning networks mutually independently judge the kind of diagnosis result to which it belongs, and then determine the final diagnosis result. For example, when the predicted result of the first dynamic learning network is positive (COVID-19) while those of 


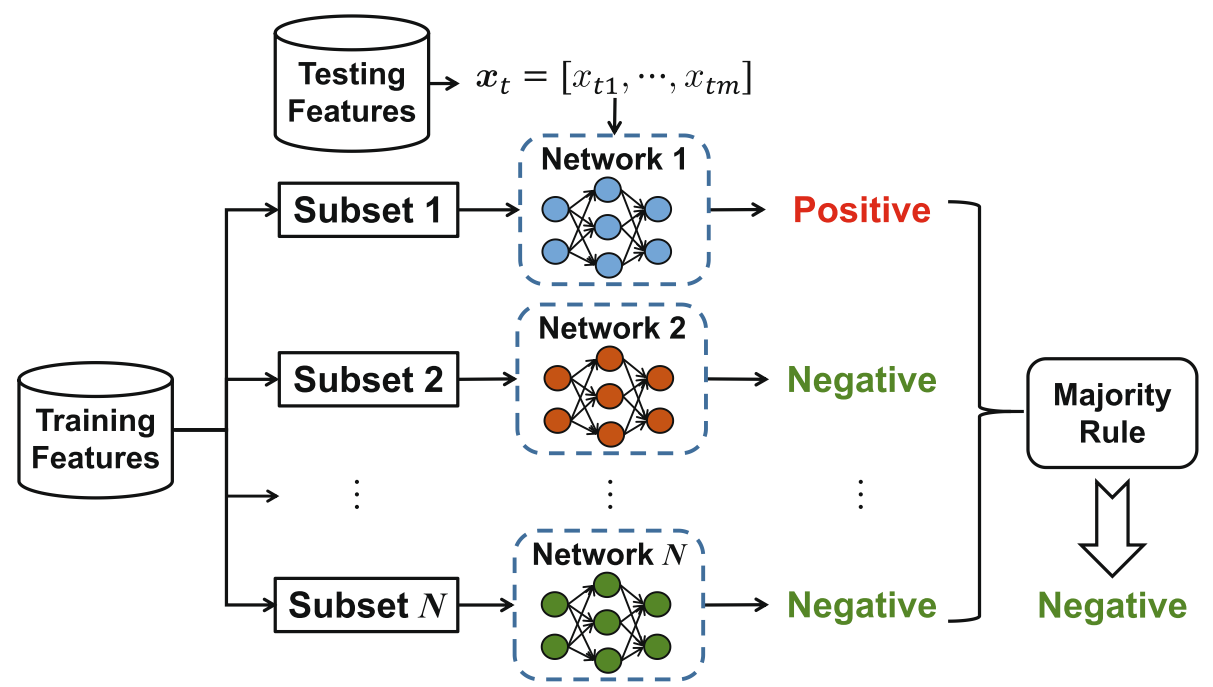

Figure 3. Concrete schematic of bagging dynamic learning network classifier in Fig. 1. $N$ dynamic learning networks trained by $N$ different types of subsets randomly under-sampled from training features predict the class to which testing images belong, and the final diagnosis result based on these predictions for the testing sample $\boldsymbol{x}_{t}=\left[x_{t 1}, \ldots, x_{t m}\right] \in \mathbb{R}^{1 \times m}$ is determined by the majority rule.

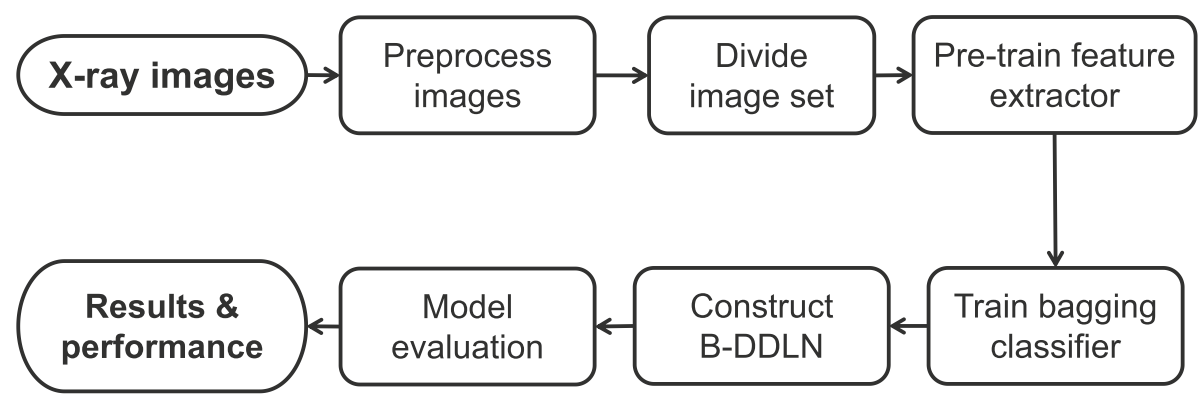

Figure 4. Flowchart of the entire experiment. Preprocessed X-ray chest radiography images are divided into training and testing sets. The training images are used to pre-train convolution blocks as a feature extractor, and a bagging dynamic learning network classifier is subsequently trained to construct B-DDLN diagnosis model. Testing images are used to evaluate the proposed B-DDLN to obtain diagnosis results and corresponding performance.

others are negative (normal), the final diagnosis result is determined as negative. If the vote is tied for a practical COVID-19 screening system, the final result is directly diagnosed as positive, which can effectively reduce the risk of missed diagnosis by enhancing the sensitivity ${ }^{43}$. When the final diagnosis result shows positive, more detailed detections and corresponding treatments must be implemented.

\section{Results}

In this section, the application of the proposed B-DDLN to diagnose the presence of COVID-19 by analyzing $\mathrm{X}$-ray chest radiography images is described. This description consists of six parts: description and preprocessing of image set, feature extraction, analysis and random under-sampling of samples, experimental results of the proposed B-DDLN, and comparisons between B-DDLN and other diagnosis models. Figure 4 shows a flowchart of the entire experiment where the feature extractor and bagging classifier are successively trained using training images and corresponding features, respectively, to construct the B-DDLN diagnosis model. Testing images are used to evaluate this proposed B-DDLN diagnosis model and the diagnosis results are obtained.

Description and preprocessing of the image set. In this study, we combine and modify the four data repositories to create the COVID-19 image set with binary-classification by leveraging the following types of patient and normal cases from each of the data repositories:

- COVID-19 patient cases from COVID-19 open image data collection, which was created by assembling medical images from websites and publications ${ }^{48}$. This data collection currently contains hundreds of frontal- 


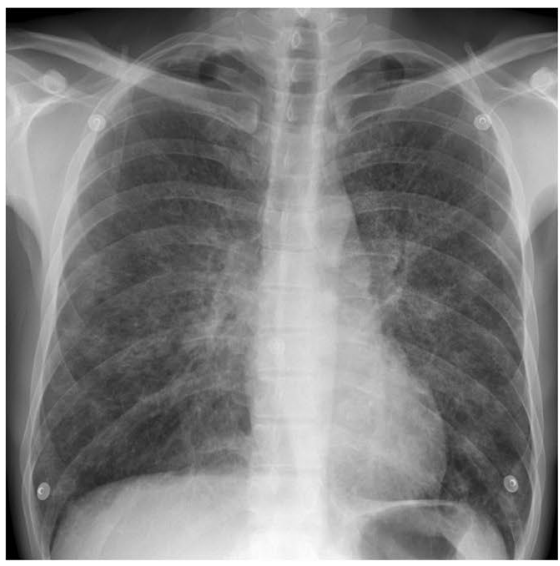

(A)

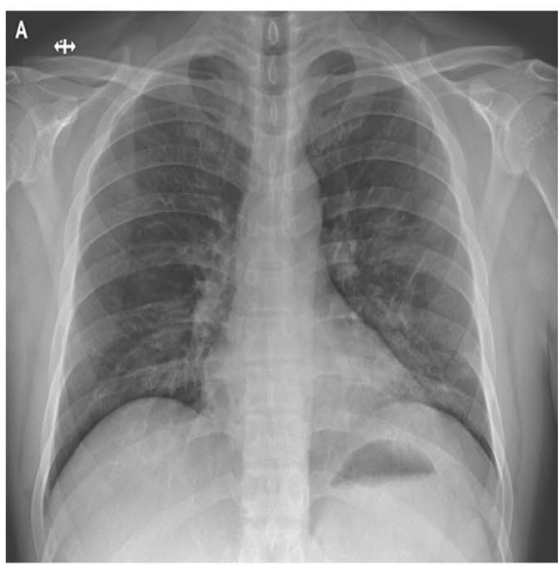

(D)

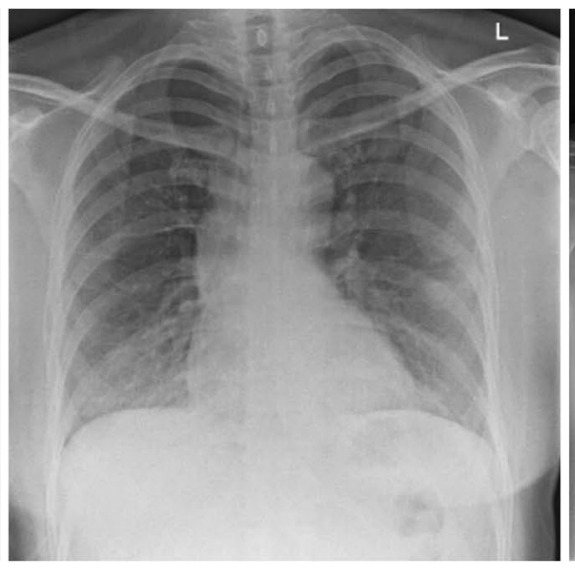

(B)

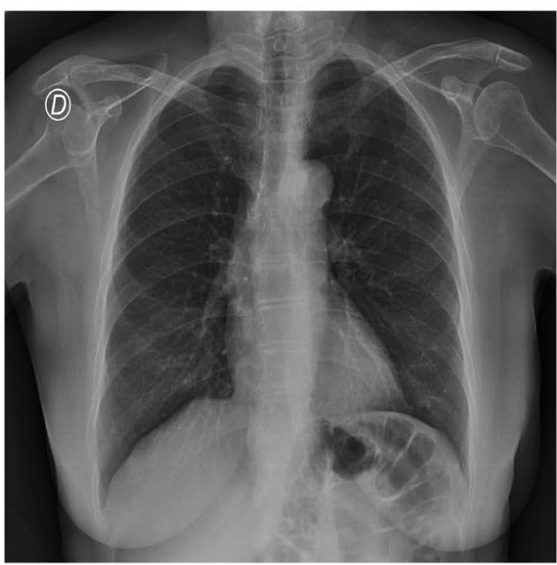

(E)

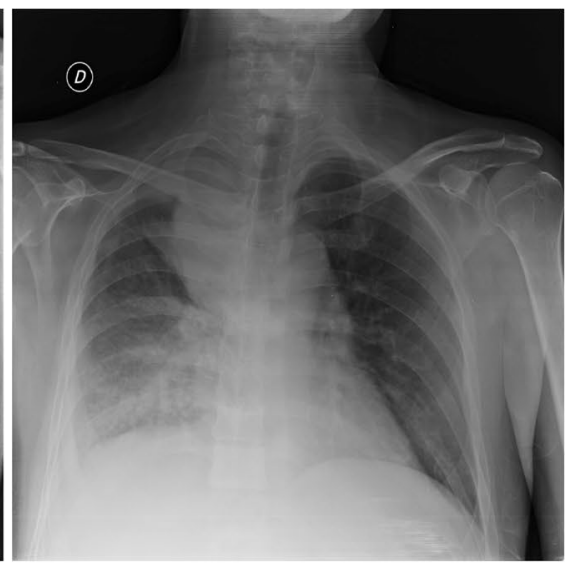

(C)

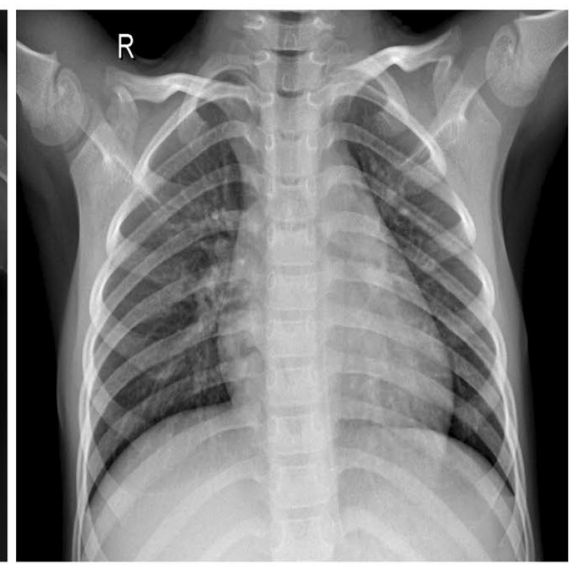

(F)

Figure 5. Examples of X-ray chest radiography images collected from COVID-19 patient and normal cases. (A) A COVID-19 patient case from COVID-19 open image data collection. (B) A COVID-19 patient case from Fig. 1 COVID-19 Chest X-ray Dataset Initiative. (C) A COVID-19 patient case from ActualMed COVID-19 chest X-ray dataset initiative. (D) A COVID-19 patient case from COVID-19 Radiography Database. (E) A normal case from ActualMed COVID-19 Chest X-ray dataset initiative. (F) A normal case from COVID-19 radiography database.

view X-ray images, which is a necessary resource to develop and evaluate tools to aid in the diagnosis of COVID-1949. An example is shown in Fig. 5A.

- COVID-19 patient cases from Fig. 1 COVID-19 Chest X-ray Dataset Initiative, which are built to enhance diagnosis models for COVID-19 disease detection and risk stratification. An example is shown in Fig. 5B.

- COVID-19 patient and normal cases from ActualMed COVID-19 Chest X-ray Dataset Initiative. A COVID19 example is shown in Fig. 5C and a normal example is shown in Fig. 5E.

- COVID-19 patient and normal cases from COVID-19 Radiography Database (winner of the COVID-19 data set award by Kaggle community), which is created by a team of researchers from Qatar University, Doha, Qatar and the University of Dhaka, Bangladesh along with their collaborators from Pakistan and Malaysia in collaboration with medical doctors. A COVID-19 example is shown in Fig. 5D and a normal example is shown in Fig. 5F.

After gathering X-ray chest radiography images from these collections, there are 2284 images in the newly formed image set (816 from COVID-19 patient cases and 1468 from normal cases). Preprocessing steps of $\mathrm{X}$-ray chest radiography images are necessary for improving the quality of image data and enhancing image features. First of all, image channel sequence is changed from BGR to RGB. Secondly, the sizes of all the images are uniformly set as $224 \times 224 \times 3$. Thirdly, these images are labeled for training B-DDLN. After the division of image set, 180 testing images (100 from normal and 80 from COVID-19 patient cases) among all are selected to evaluate the proposed B-DDLN.

Feature extractor pre-training. The feature extractor of images is pre-trained in an environment equipped with PyCharm Community Edition of 2020.1.2 version and MATLAB 2020a on a personal computer with an Intel Core i7 processor, NVIDIA GeForce GTX 1660 Ti GPU architecture and 32 GB RAM running under Windows 10 operating system. We adopt scikit-learn, TensorFlow 2.2.1 and keras to build convolution 


\begin{tabular}{|l|l|l|l|l|l|l|}
\hline Dimension & Variation source & Square sum $(\mathbf{S S})$ & Degree of freedom $(\mathbf{d f})$ & $\mathbf{S S} / \mathbf{d f}$ & $\boldsymbol{F}$-value & $\boldsymbol{p}$-value \\
\hline \multirow{4}{*}{1 st } & Groups & 55.563 & 1 & 55.6625 & 2155.96 & 0 \\
\cline { 2 - 8 } & Error & 54.269 & 2102 & 0.0258 & - & - \\
\cline { 2 - 8 } & Total & 109.932 & 2103 & - & - & - \\
\hline \multirow{3}{*}{ 2nd } & Groups & 2.2625 & 1 & 2.2625 & 160.75 & $1.49852 \times 10^{-35}$ \\
\cline { 2 - 8 } & Error & 29.5846 & 2102 & 0.01407 & - & - \\
\cline { 2 - 8 } & Total & 31.8471 & 2103 & - & - & - \\
\hline \multirow{3}{*}{3 rd } & Groups & 39.553 & 1 & 39.5534 & 773.36 & $3.35758 \times 10^{-145}$ \\
\cline { 2 - 8 } & Error & 107.506 & 2102 & -0.0511 & - & - \\
\cline { 2 - 8 } & Total & 147.06 & 2103 & - & - & - \\
\hline
\end{tabular}

Table 1. Analytic results among training feature samples in the previous three dimensions by one-way ANOVA.

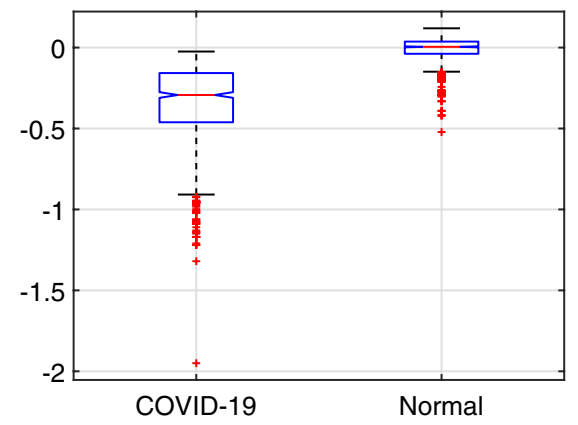

(A)

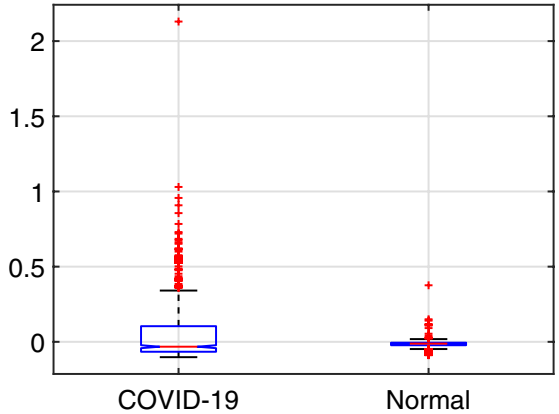

(B)

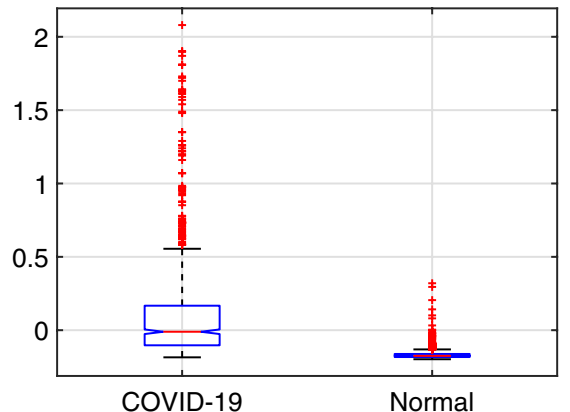

(C)

Figure 6. Box-plots among training feature samples in the previous three dimensions by one-way ANOVA. (A) The 1st dimension. (B) The 2nd dimension. (C) The 3rd dimension.

blocks and pre-train them. When these convolution blocks finish pre-training as a feature extractor $h(\cdot)$, a $224 \times 224 \times 3$ preprocessed X-ray chest radiography image tensor $\boldsymbol{g}_{s}$ is input into $h(\cdot)$, and its corresponding 512-dimension extracted feature vector $h\left(\boldsymbol{g}_{s}\right)$ can be obtained as output. Therefore, the features extracted from training images are utilized to train the bagging dynamic learning network classifier, and the features extracted from testing images are used to evaluate this bagging dynamic learning network classifier.

One-way analysis of variance. To investigate whether different values of an attribute have a significant effect on the category, one-way analysis of variance (one-way ANOVA) is carried out by using the features extracted from training images in this study. Each training feature sample is a 512-dimension vector. The analytic results of the previous three dimensions by one-way ANOVA are obtained as examples, which are listed in Table 1. Corresponding box-plots are shown in Fig. 6. It can be seen from Table 1 that the $p$-value is much smaller than 0.05 , which suggests that a correlation exists between these three dimensions of features and sample category. The results of $F$-value and $p$-value in Table 1 and Fig. 6 collectively substantiate the existence of significant differences among training feature samples belonging to different categories.

Random under-sampling of image features. After division of the image set, there are 2104 left in the training images, among which 736 come from COVID-19 patient and 1368 from normal cases. Such distribution causes category imbalance, which hinders the classifier from releasing effective performance. To overcome this problem, random under-sampling of samples (i.e., feature vectors) is necessary, which means that 1000 samples from COVID-19 patient cases are randomly selected among all. In this study, we perform this random under-sampling three times and obtain three different subsets. Each subset consists of 1736 samples, with 736 come from COVID-19 patient cases and 1000 from normal cases. By using these three subsets, three dynamic learning networks (i.e., $N=3$ ) with three different types of mapping functions are trained. As examples, these three types of mapping functions are: linear (i.e., Eq. (14)), tanh (i.e., Eq. (15)) and sinh (i.e., Eq. (16)) types. Furthermore, the bagging dynamic learning network classifier is constructed by these three dynamic learning networks and bagging algorithm.

Classification performance of B-DDLN. In this study, two cases of activation functions are designed in the dynamic learning network. In case 1, all the activation functions in the hidden and output neurons are set as softsign function (i.e., Eq. (6)). In case 2, activation functions in the output neurons are also designed as softsign function while those in the hidden neurons are set as power-softsign function (i.e., Eq. (7)). For classifying the extracted features from convolution blocks of B-DDLN, trends of classification errors calculated according to 


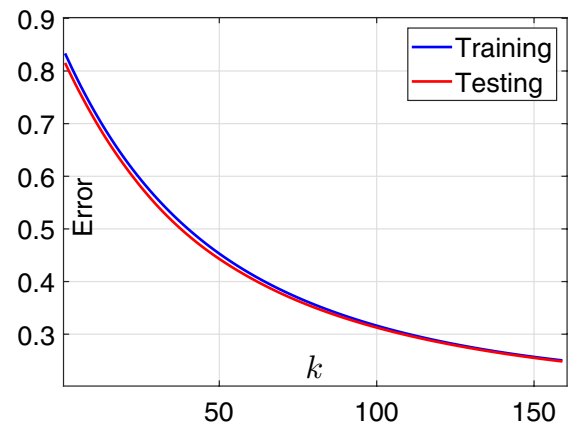

(A)

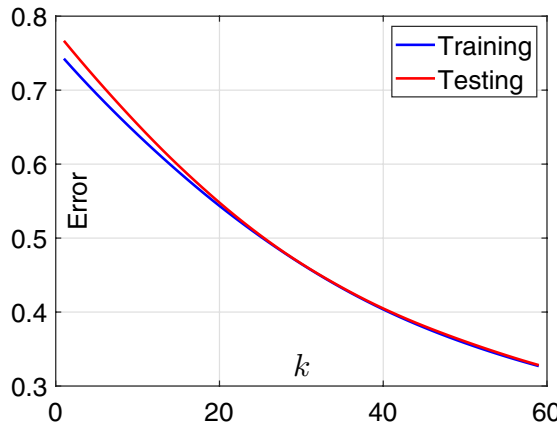

(B)

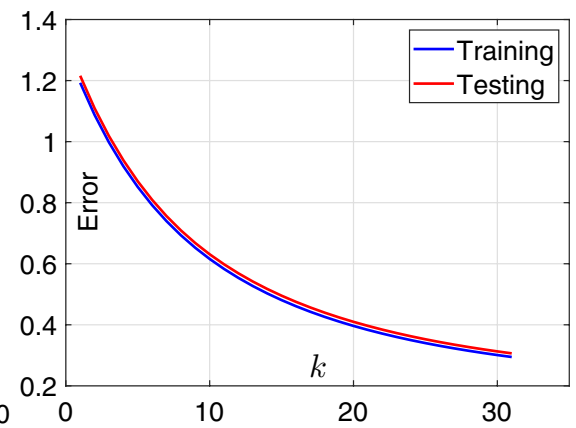

(C)

Figure 7. Trends of errors for training and testing dynamic learning network (DLN) in case 1 as the training round $k$ increases. (A) DLN trained by NDLA with linear type of mapping function. (B) DLN trained by NDLA with tanh type of mapping function. (C) DLN trained by NDLA with sinh type of mapping function.

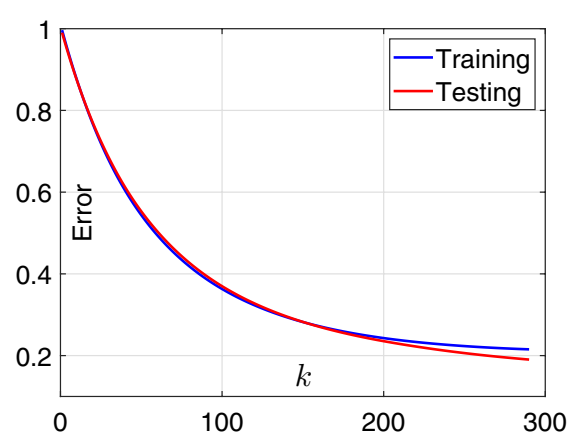

(A)

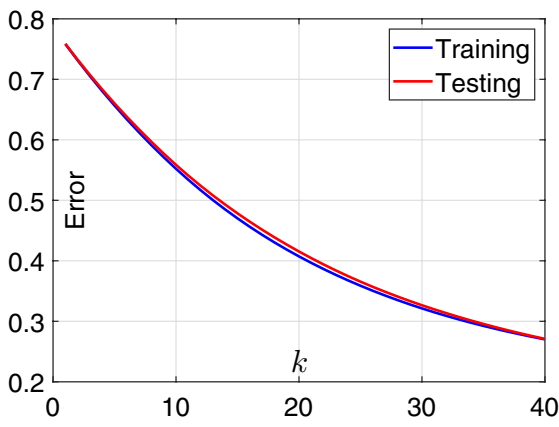

(B)

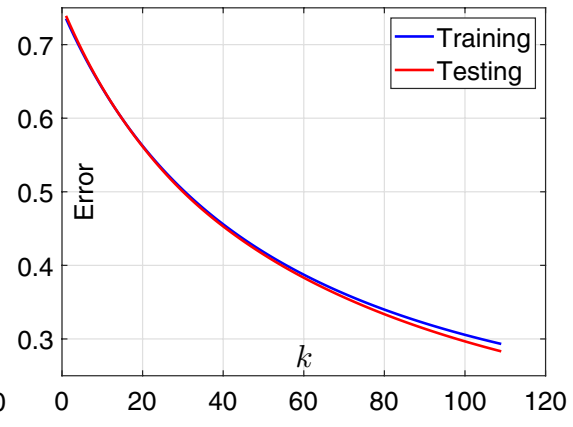

(C)

Figure 8. Trends of errors for training and testing dynamic learning network (DLN) in case 2 as the training round $k$ increases. (A) DLN trained by NDLA with linear type of mapping function. (B) DLN trained by NDLA with tanh type of mapping function. (C) DLN trained by NDLA with sinh type of mapping function.

\begin{tabular}{|l|l|l|l|l|}
\hline Activation function & Model & \multicolumn{2}{|l|}{ Confusion matrix } & \multirow{2}{*}{ Accuracy (\%) } \\
\hline \multirow{2}{*}{ Case 1 } & \multirow{2}{*}{ B-DDLN } & $\mathrm{TP}=99$ & $\mathrm{FN}=1$ & \multirow{2}{*}{98.8889} \\
\cline { 3 - 4 } & & $\mathrm{FP}=1$ & $\mathrm{TN}=79$ & \\
\hline \multirow{2}{*}{ Case 2 } & \multirow{2}{*}{ B-DDLN } & $\mathrm{TP}=99$ & $\mathrm{FN}=1$ & \multirow{2}{*}{98.8889} \\
\cline { 3 - 4 } & & $\mathrm{FP}=1$ & $\mathrm{TN}=79$ & \\
\hline
\end{tabular}

Table 2. Confusion matrices of the proposed B-DDLN.

cross entropy formula (i.e., Eq. (18)) of dynamic learning networks trained by NDLA in two cases are shown in Figs. 7 and 8 . From Figs. 7 and 8 , we can see that both two tendencies monotonically and rapidly decrease and even converge to a specific minor value when the training round $k$ is large enough. The testing errors $\varepsilon_{t}$ in all of the subgraphs are always decreasing as $k$ increases. These phenomena indicate that the dynamic learning networks trained by NDLA with different mapping and activation functions are not over-fitting. They also verify that using NDLA can rapidly make the classification error monotonously converge and enhance precision. In the testing process, confusion matrices of the proposed B-DDLNs under both two cases of activation functions are listed in Table 2, where

True (T): Actual result is normal.

False (F): Actual result is COVID-19.

Positive (P): Predicted result is normal.

Negative (N): Predicted result is COVID-19.

Thus, the testing accuracy is calculated by Eq. (27), i.e., 


\begin{tabular}{|l|l|l|l|l|}
\hline \multirow{4}{*}{ Activation function } & & \multicolumn{3}{|l|}{ Mapping function type } \\
\cline { 2 - 5 } & Accuracy and hyper-parameters & Linear & Tanh & Sinh \\
\hline \multirow{5}{*}{ Case 1 } & Testing accuracy $(\%)$ & 98.3333 & 97.2222 & 96.6667 \\
\cline { 2 - 5 } & Training time $(s)$ & 6.0156 & 2.3906 & 1.4688 \\
\cline { 2 - 5 } & Threshold $\varepsilon^{\prime}$ & 0.2506 & 0.3270 & 0.2947 \\
\cline { 2 - 5 } & Training rounds $k$ & 159 & 59 & 31 \\
\cline { 2 - 5 } & NDLA coefficient $\alpha$ & 0.01 & 0.02 & 0.04 \\
\cline { 2 - 5 } & Hidden neurons $n$ & 50 & 55 & 60 \\
\hline \multirow{5}{*}{ Case 2 } & Testing Accuracy $(\%)$ & 98.3333 & 97.2222 & 97.7778 \\
\cline { 2 - 5 } & Training time $(s)$ & 17.5000 & 2.7344 & 6.2344 \\
\cline { 2 - 5 } & Threshold $\varepsilon^{\prime}$ & 0.2154 & 0.2711 & 0.2935 \\
\cline { 2 - 5 } & Training rounds $k$ & 290 & 40 & 109 \\
\cline { 2 - 5 } & NDLA coefficient $\alpha$ & 0.01 & 0.04 & 0.01 \\
\cline { 2 - 5 } & Hidden neurons $n$ & 42 & 40 & 41 \\
\hline
\end{tabular}

Table 3. Accuracies and hyper-parameters of single dynamic learning networks.

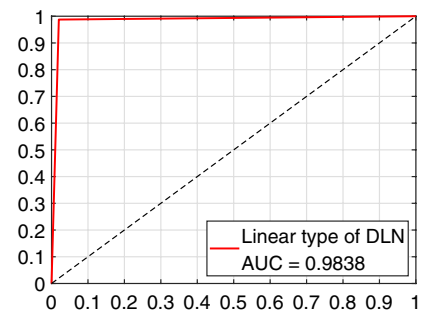

(A)

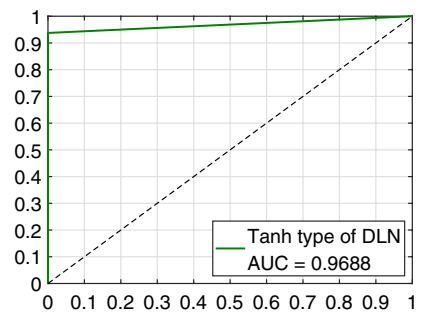

(B)

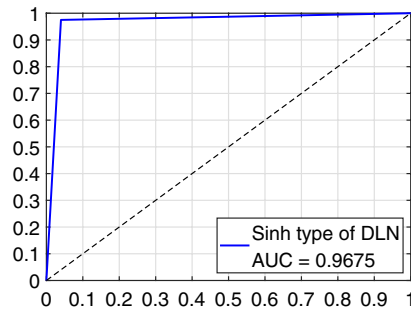

(C)

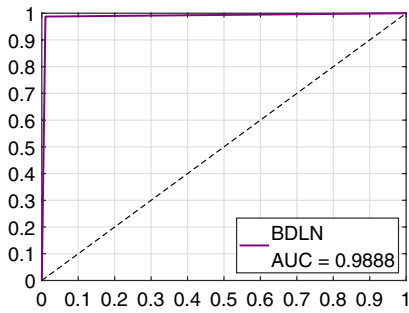

(D)

Figure 9. Receiver operating characteristic curves (ROC curves) of dynamic learning networks (DLN) and bagging dynamic learning network (BDLN) in case 1. (A) DLN trained by NDLA with linear type of mapping function. (B) DLN trained by NDLA with tanh type of mapping function. (C) DLN trained by NDLA with sinh type of mapping function. (D) Bagging dynamic learning network (BDLN) classifier.

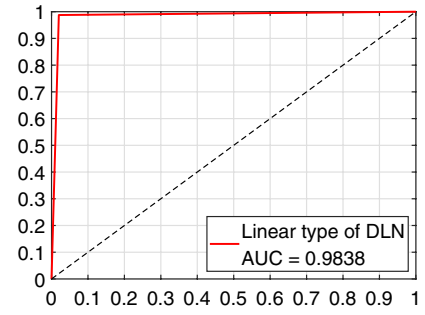

(A)

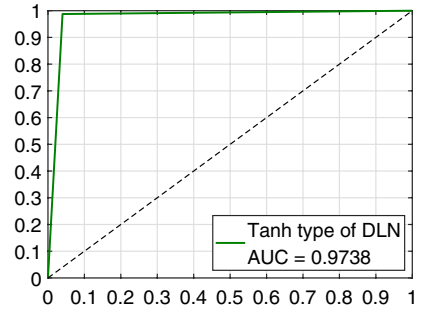

(B)

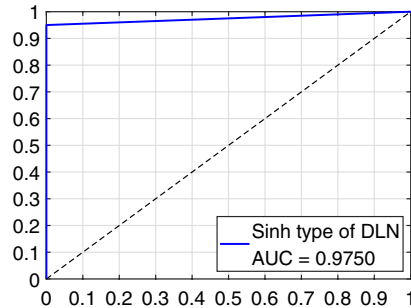

(C)

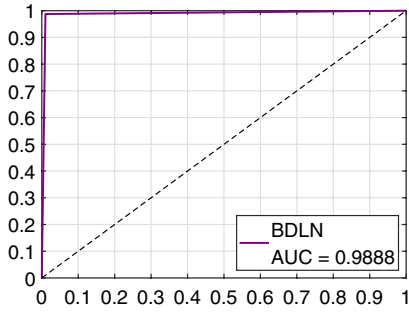

(D)

Figure 10. Receiver operating characteristic curves (ROC curves) of dynamic learning networks (DLN) and bagging dynamic learning network (BDLN) in case 2. (A) DLN trained by NDLA with linear type of mapping function. (B) DLN trained by NDLA with tanh type of mapping function. (C) DLN trained by NDLA with sinh type of mapping function. (D) Bagging dynamic learning network (BDLN) classifier.

$$
\text { Accuracy }=\frac{\mathrm{TP}+\mathrm{TN}}{\mathrm{TP}+\mathrm{TN}+\mathrm{FP}+\mathrm{FN}} .
$$

In Table 2, most testing samples distribute in the TP and TN areas, which illustrates that the proposed B-DDLNs in two cases achieve high diagnosis accuracy. The accuracies of dynamic learning networks with corresponding hyper-parameters in two cases are listed in Table 3 . In the process of training the proposed dynamic learning network, the proper sets of hyper-parameters are adjusted and selected by 10 -fold cross validation, which makes the testing performance including accuracy reach the best level. Early stopping is also adopted, which can avoid over-fitting. From Table 3, we can see that the highest testing accuracy of a single dynamic learning network can reach $98.3333 \%$ in a short time. After plurality voting, the bagging dynamic learning networks in two cases achieve $98.8889 \%$ accuracy, which shows good precision for diagnosing COVID-19. The number of hidden neurons is no more than 60 , which suggests that the dynamic learning network is a lightweight network. 


\begin{tabular}{|c|c|}
\hline Model/method & Testing accuracy $(\%)$ \\
\hline The proposed B-DDLN (case 1) & 98.8889 \\
\hline The proposed B-DDLN (case 2) & 98.8889 \\
\hline ResNet & 97.2222 \\
\hline CNN-X (transfer learning) ${ }^{16}$ & 95.0000 \\
\hline ResNet-based multi-channel transfer learning model ${ }^{50}$ & 93.8889 \\
\hline DarkCovidNet $^{51}$ & 97.7778 \\
\hline SPEA-II-based modified AlexNet ${ }^{52}$ & 98.3333 \\
\hline Ensemble densely connected convolutional neural network ${ }^{53}$ & 97.2222 \\
\hline Ensemble deep transfer learning model ${ }^{54}$ & 96.1111 \\
\hline
\end{tabular}

Table 4. Comparison results between B-DDLN and some existing state-of-the-art methods. The superior performance of the proposed B-DDLN diagnosis model are highlighted in bold.

\begin{tabular}{|l|l|l|l|l|l|l|l|}
\hline Classifier & Testing accuracy(\%) & F1-score & Precision & Specificity & Sensitivity & Kappa value & AUC \\
\hline B-DDLN1 $^{\text {a }}$ & $\mathbf{9 8 . 8 8 8 9}$ & $\mathbf{0 . 9 9 0 0}$ & $\mathbf{0 . 9 9 0 0}$ & $\mathbf{0 . 9 8 7 5}$ & $\mathbf{0 . 9 9 0 0}$ & $\mathbf{0 . 9 7 7 5}$ & $\mathbf{0 . 9 8 8 8}$ \\
\hline B-DDLN2 $^{\text {b }}$ & $\mathbf{9 8 . 8 8 8 9}$ & $\mathbf{0 . 9 9 0 0}$ & $\mathbf{0 . 9 9 0 0}$ & $\mathbf{0 . 9 8 7 5}$ & $\mathbf{0 . 9 9 0 0}$ & $\mathbf{0 . 9 7 7 5}$ & $\mathbf{0 . 9 8 8 8}$ \\
\hline BPNN $^{\text {c }}$ & 95.5556 & 0.9588 & 0.9894 & 0.9875 & 0.9300 & 0.9107 & 0.9587 \\
\hline Naive Bayesian & 92.7778 & 0.9353 & 0.9307 & 0.9125 & 0.9400 & 0.8536 & 0.9012 \\
\hline LDA $^{\text {d }}$ & 93.3333 & 0.9406 & 0.9314 & 0.9125 & 0.9500 & 0.8647 & 0.9313 \\
\hline QDA $^{\text {e }}$ & 94.4444 & 0.9485 & 0.9787 & 0.9750 & 0.9200 & 0.8883 & 0.9475 \\
\hline Linear SVM & 96.6667 & 0.9697 & 0.9796 & 0.9750 & 0.9600 & 0.9327 & 0.9675 \\
\hline Gaussian SVM & 95.5556 & 0.9600 & 0.9600 & 0.9500 & 0.9600 & 0.9100 & 0.9550 \\
\hline$k$-NN & 95.0000 & 0.9569 & 0.9174 & 0.8875 & 1.0000 & 0.8976 & 0.9437 \\
\hline Logistic regression & 90.5556 & 0.9171 & 0.8952 & 0.8625 & 0.9400 & 0.8075 & 0.9012 \\
\hline Decision tree & 93.3333 & 0.9394 & 0.9490 & 0.9375 & 0.9300 & 0.8653 & 0.9337 \\
\hline Bagging tree & 92.7778 & 0.9366 & 0.9143 & 0.8875 & 0.9600 & 0.8528 & 0.9237 \\
\hline Boosting tree & 96.1111 & 0.9648 & 0.9697 & 0.9625 & 0.9600 & 0.9213 & 0.9612 \\
\hline
\end{tabular}

Table 5. Comparison results between B-DDLN and other classifiers for classifying the same extracted image features. The superior performance of the proposed B-DDLN diagnosis model are highlighted in bold. ${ }^{\mathrm{a}} \mathrm{B}-\mathrm{DDLN}$ in case $1 .{ }^{\mathrm{b}} \mathrm{B}-\mathrm{DDLN}$ in case $2 .{ }^{\mathrm{c}} \mathrm{B}$ ack propagation neural network. ${ }^{\mathrm{d}}$ Linear discriminant analysis. ${ }^{\mathrm{e}} \mathrm{Quadratic}$ discriminant analysis. ${ }^{\mathrm{f}}$ Support vector machine. ${ }^{\mathrm{g}} \mathrm{k}$-nearest neighbor.

For the testing set, the receiver operating characteristic curves (ROC curves) of the dynamic learning network and its improved models, i.e., bagging dynamic learning network classifiers, under two cases are shown in Figs. 9 and 10 . It can be seen that these ROC curves approach coordinate point $(0,1)$, which leads to large corresponding areas under curves (AUC). These phenomena further substantiate the superior classification performance of the proposed B-DDLN diagnosis model.

Comparison analysis. In order to further verify the superiority of the proposed B-DDLN diagnosis model, fair and comprehensive comparisons in classification performance between B-DDLN and other existing methods on the same image set are presented in this section.

For comparisons on the same application domain, we use the same images and 10-fold cross validation to train some state-of-the-art methods under the same operating environment, and we apply the same testing images to evaluate them. Testing accuracy is used as the main indicator to measure the classification performance of deep learning models, whose results are listed in Table 4. In Table 4, testing accuracy of the proposed B-DDLN is an average value, while those of others show the highest values. From Table 4, we can see that the testing accuracy of the proposed B-DDLN reaches the top level when compared with other deep learning models, which manifests the superior precision of the proposed B-DDLN in diagnosing COVID-19. In addition, the structure of proposed B-DDLN is simpler and more lightweight than most of the existing state-of-the-art models, which suggests that B-DDLN is more easily deployed in a medical software system with a higher speed of diagnostic prediction.

For the same extracted image features, comparison results in classification performance between the proposed B-DDLN, especially its bagging dynamic learning network, and other classifiers are listed in Table 5, where the corresponding experiments are conducted under the same conditions. From Table 5, we can see that the specificity and sensitivity of the proposed B-DDLN are 0.9875 and 0.9900 , respectively, which indicates that misdiagnosis and missed diagnosis of COVID-19 are very rare. Most of the evaluation indicators simultaneously remain the highest among all the classifiers. These findings substantiate the excellent advantages and superiorities of the proposed B-DDLN in diagnosing the presence of COVID-19. 


\section{Discussion}

From the experimental and compared results, we can see that using the proposed B-DDLN, which consists of a feature extractor and a bagging dynamic learning network classifier, achieves the best diagnostic performance compared with other state-of-the-art models on the same application domain. The proposed B-DDLN is trained and constructed in a short time, and shows superior classification performance without over-fitting. The diagnosis accuracy for distinguishing COVID-19 or normal stays at the top level when using the proposed B-DDLN owing to the existence of NDLA and bagging algorithm. The proposed B-DDLN diagnosis model is so lightweight that it can be deployed as a medical software system under the restrictions of high real-time requirement and limited hardware resources.

However, the proposed B-DDLN has certain limitations, and some improvements can be made. First of all, the extracted features of X-ray chest radiography images are not detailed and separable enough. The feature extractor needs to be improved by remolding and optimizing the structure and pre-training method. Secondly, the forms of labels for input images, i.e., $\overline{\boldsymbol{Y}}$ and $\boldsymbol{L}$, are not unified, which impedes training dynamic learning networks. Thirdly, the calculation method of class possibility needs to be improved to enhance confidence. Furthermore, the proposed B-DDLN should be used for diagnosing different types of diseases to verify its generalization performance.

\section{Conclusion}

In this paper, B-DDLN has been proposed for diagnosing presence of COVID-19 using X-ray chest radiography images. After some preprocessing steps, training images have been used to pre-train convolution blocks as a feature extractor. For the training features extracted from this extractor, a bagging dynamic learning network classifier based on neural dynamic learning algorithm (NDLA) and bagging algorithm has been trained. Experimental results have verified that the proposed B-DDLN diagnosis model possesses high training efficiency, and has considerably increased diagnosis accuracy under the restrictions of high real-time requirement and limited hardware resources. The diagnosis accuracy of the proposed B-DDLN has reached $98.8889 \%$, which is the highest among all the existing state-of-the-art methods. In addition, corresponding performance indicators of bagging dynamic learning network classifier have reached the top level among all, further substantiating that the proposed B-DDLN possesses excellent diagnostic ability. Such accurate diagnosis outcomes provide assertive evidences in early detection of COVID-19, which helps to provide suitable treatments for patients and maintain human health.

In the future, the proposed B-DDLN diagnosis model can be trained for diagnosing not only the presence of COVID-19, but also other types of pneumonia such as SARS and community-acquired pneumonia, which cannot be detected by nucleic acid amplification testing alone. For excellent diagnostic performance, a medical software system based on the proposed B-DDLN diagnosis model can be developed for diagnosing multi-classification of pneumonia by automatically recognizing X-ray chest radiography images, generating diagnostic reports, and providing corresponding therapeutic schemes for diagnostic results. Such a fully functional system would be extremely useful for clinicians.

\section{Data availability}

COVID-19 image data collection is available at https://github.com/ieee8023/covid-chestxray-dataset. Figure 1 COVID-19 chest X-ray dataset initiative is available at https://github.com/agchung/Figure1-COVID-chestxraydataset. Actualmed COVID-19 chest X-ray dataset initiative is available at https://github.com/agchung/Actua lmed-COVID-chestxray-dataset. COVID-19 radiography database is available at https://www.kaggle.com/tawsi furrahman/covid19-radiography-database.

\section{Code availability}

Source codes and data set of the current study are available at https://github.com/OliverChan96/B-DDLN.

Received: 11 April 2021; Accepted: 26 July 2021

Published online: 11 August 2021

\section{References}

1. Nishio, M., Noguchi, S., Matsuo, H. \& Murakami, T. Automatic classification between COVID-19 pneumonia, non-COVID-19 pneumonia, and the healthy on chest X-ray image: Combination of data augmentation methods. Sci. Rep. 10, 17532 (2020).

2. Luengo-Oroz, M. et al. Artificial intelligence cooperation to support the global response to COVID-19. Nat. Mach. Intell. 2, 295-297 (2020)

3. Singh, D., Vijay Kumar, V. \& Kaur, M. Classification of COVID-19 patients from chest CT images using multi-objective differential evolution-based convolutional neural networks. Eur. J. Clin. Microbiol. Infect. Dis. 39, 1379-1389 (2020).

4. Wilson, I. G. Inhibition and facilitation of nucleic acid amplification. Appl. Environ. Microbiol. 63, 3741-3751 (1997).

5. Singh, K. K. \& Singh, A. Diagnosis of COVID-19 from chest X-Ray Images using wavelets-based depthwise convolution network. Big Data Min. Anal. 4, 84-93 (2021).

6. Yan, L. et al. An interpretable mortality prediction model for COVID-19 patients. Nat. Mach. Intell. 2, 283-288 (2020).

7. Ai, T. et al. Correlation of chest CT and RT-PCR testing for coronavirus disease 2019 (COVID-19) in China: A report of 1014 cases. Radiology 296, e32-e40 (2020).

8. Korot, E. et al. Code-free deep learning for multi-modality medical image classification. Nat. Mach. Intell. 3, 288-298 (2021).

9. Sauter, A. P. et al. Optimization of tube voltage in X-ray dark-field chest radiography. Sci. Rep. 9, 8699 (2019).

10. Tabik, S. et al. COVIDGR dataset and COVID-SDNet methodology for predicting COVID-19 based on chest X-Ray images. IEEE J. Biomed. Health Inform. 24, 3595-3605 (2020).

11. Shi, F. et al. Review of artificial intelligence techniques in imaging data acquisition, segmentation, and diagnosis for COVID-19. IEEE Rev. Biomed. Eng. 14, 4-15 (2021).

12. Liang, T. Handbook of COVID-19 Prevention and Treatment (School of Medicine Zhejiang University, China, The First Affiliated Hospital, 2020). 
13. Wang, Z. et al. Automatically discriminating and localizing COVID-19 from community-acquired pneumonia on chest X-rays. Pattern Recognit. 110, 107613 (2021).

14. Kumar, V., Singh, D., Kaur, M. \& Damaševičius, D. Overview of current state of research on the application of artificial intelligence techniques for COVID-19. PeerJ Comput. Sci. 7, e564 (2021).

15. Zhang, J. et al. Viral pneumonia screening on chest X-rays using confidence-aware anomaly detection. IEEE Trans. Med. Imaging 40, 879-890 (2021).

16. Majeed, T., Rashid, R., Ali, D. \& Asaad, A. COVID-19 detection using CNN transfer learning from X-ray images. medRxiv Preprint. https://doi.org/10.1101/2020.05.12.20098954 (2020).

17. Singh, D., Kumar, V., Yadav, V. \& Kaur, M. Deep neural network-based screening model for COVID-19-infected patients using chest X-Ray images. Int. J. Pattern Recognit. Artif. Intell. 35, 2151004 (2021).

18. Brunese, L., Mercaldo, F., Reginelli, A. \& Santone, A. Explainable deep learning for pulmonary disease and coronavirus COVID-19 detection from X-rays. Comput. Meth. Programs Biomed. 196, 105608 (2020).

19. LeCun, Y., Bengio, Y. \& Hinton, G. Deep learning. Nature 521, 436-444 (2015).

20. He, K., Zhang, X., Ren, S. \& Sun, J. Deep residual learning for image recognition. In Proc. IEEE Comput. Soc. Conf. Comput. Vision Pattern Recognit. (CVPR) 770-778 (IEEE, 2016).

21. Bressem, K. K. et al. Comparing different deep learning architectures for classification of chest radiographs. Sci. Rep. 10, 13590 (2020).

22. Dai, X., Wu, X., Wang, B. \& Zhang, L. Semisupervised scene classification for remote sensing images: A method based on convolutional neural networks and ensemble learning. IEEE Geosci. Remote Sens. Lett. 16, 869-873 (2019).

23. Luz, E. et al. Towards an effective and efficient deep learning model for COVID-19 patterns detection in X-ray images. Res. Biomed. Eng. https://doi.org/10.1007/s42600-021-00151-6 (2021).

24. Farooq, M. \& Hafeez, A. COVID-ResNet: A deep learning framework for screening of COVID19 from radiographs. arXiv preprint. arxiv:2003.14395 (2020).

25. Huang, G., Liu, Z., van der Maaten, L. \& Weinberger, K. Q. Densely connected convolutional networks. in Proceedings of the IEEE Computer Society Conference on Computer Vision and Pattern Recognition (CVPR) 2261-2269 (IEEE, 2017).

26. Wang, L., Lin, Z. Q. \& Wong, A. COVID-Net: A tailored deep convolutional neural network design for detection of COVID-19 cases from chest X-ray images. Sci. Rep. 10, 19549 (2020).

27. Narayan Das, N., Kumar, N., Kaur, M., Kumar, V. \& Singh, D. Automated deep transfer learning-based approach for detection of COVID-19 infection in chest X-rays. IRBM. https://doi.org/10.1016/j.irbm.2020.07.001 (2020).

28. Zeng, Y., Xu, X., Shen, D., Fang, Y. \& Xiao, Z. Traffic sign recognition using kernel extreme learning machines with deep perceptual features. IEEE Trans. Intell. Transp. Syst. 18, 1647-1653 (2017).

29. Sethy, P. K., Behera, S. K., Ratha, P. K. \& Biswas, P. Detection of coronavirus disease (COVID-19) based on deep features and support vector machine. Int. J. Math. Eng. Manage. Sci. 5, 643-651 (2020).

30. Novitasari, D. C. R. et al. Detection of COVID-19 chest X-ray using support vector machine and convolutional neural network. Commun. Math. Biol. Neurosci. 2020, 1-19 (2020).

31. Zhang, Z. et al. A new varying-parameter convergent-differential neural-network for solving time-varying convex QP problem constrained by linear-equality. IEEE Trans. Autom. Control 63, 4110-4125 (2018).

32. Zhang, Z. et al. A new varying-parameter recurrent neural-network for online solution of time-varying Sylvester equation. IEEE Trans. Cybern. 48, 3135-3148 (2018).

33. Zhang, Z., Zheng, L., Qiu, T. \& Deng, F. Varying-parameter convergent-differential neural solution to time-varying overdetermined system of linear equations. IEEE Trans. Autom. Control 65, 874-881 (2020).

34. Zhang, Z. \& Zheng, L. A complex varying-parameter convergent-differential neural-network for solving online time-varying complex Sylvester equation. IEEE Trans. Cybern. 49, 3627-3639 (2019).

35. Zhang, Z., Chen, T., Wang, M. \& Zheng, L. An exponential-type anti-noise varying-gain network for solving disturbed time-varying inversion systems. IEEE Trans. Neural Netw. Learn. Syst. 31, 3414-3427 (2020).

36. Zhang, Z. \& Yan, Z. A varying parameter recurrent neural network for solving nonrepetitive motion problems of redundant robot manipulators. IEEE Trans. Control Syst. Technol. 27, 2680-2687 (2019).

37. Zhang, Z. et al. A varying-parameter convergent-differential neural network for solving joint-angular-drift problems of redundant robot manipulators. IEEE/ASME Trans. Mechatron. 23, 679-689 (2018).

38. Zhang, Z. et al. A varying-parameter adaptive multi-layer neural dynamic method for designing controllers and application to unmanned aerial vehicles. IEEE Trans. Intell. Transp. Syst. https://ieeexplore.ieee.org/document/9072299 (2020).

39. Zheng, L. \& Zhang, Z. Convergence and robustness analysis of novel adaptive multilayer neural dynamics-based controllers of multirotor UAVs. IEEE Trans. Cybern. 51, 3710-3723 (2021).

40. Zhang, Z., Kong, L. \& Zheng, L. Power-type varying-parameter RNN for solving TVQP problems: Design, analysis, and applications. IEEE Trans. Neural Netw. Learn. Syst. 30, 2419-2433 (2019).

41. Zhang, Z. et al. Robustness analysis of a power-type varying-parameter recurrent neural network for solving time-varying QM and QP problems and applications. IEEE Trans. Syst. Man Cybern. A Syst. 50, 5106-5118 (2020).

42. Zhang, Z. \& Yan, Z. An adaptive fuzzy recurrent neural network for solving the nonrepetitive motion problem of redundant robot manipulators. IEEE Trans. Fuzzy Syst. 28, 684-691 (2020).

43. Zhang, Z., Chen, B., Xu, S., Chen, G. \& Xie, J. A novel voting convergent difference neural network for diagnosing breast cancer. Neurocomputing 437, 339-350 (2021).

44. Theodoridis, S. \& Koutroumbas, K. Pattern Recognition, Ch. 2 4th edn. (Academic Press, 2009).

45. Xiao, L. \& Zhang, Y. Two new types of Zhang neural networks solving systems of time-varying nonlinear inequalities. IEEE Trans. Circuits Syst. I-Regul. Pap. 59, 2363-2373 (2012).

46. Zhang, Y., Xiao, H., Wang, J., Li, J. \& Chen, P. Discrete-time control and simulation of ship course tracking using ZD method and ZFD formula 4NgSFD. in Proceedings of the IEEE 3rd Information Technology and Mechatronics Engineering Conference (ITOEC) 6-10 (IEEE, 2017).

47. Oza, N. C. \& Russhell, S. Experimental comparisons of online and batch versions of bagging and boosting. in Proceedings of the Seventh ACM SIGKDD International Conference on Knowledge Discovery and Data Mining 359-364 (ACM, 2001)

48. Cohen, J. P., Morrison, P. \& Dao, L. COVID-19 image data collection. arXiv preprint. arxiv:2003.11597 (2020).

49. Cohen, J. P. et al. COVID-19 image data collection: Prospective predictions are the future. arXiv preprint. arxiv:2006.11988 v3 (2020).

50. Misra, S., Jeon, S., Lee, S., Managuli, R. \& Kim, C. Multi-channel transfer learning of chest X-ray images for screening of COVID19. Electronics 9,1388 (2020).

51. Ozturk, T. et al. Automated detection of COVID-19 cases using deep neural networks with X-ray images. Comput. Biol. Med. 121, $103792(2020)$.

52. Kaur, M. et al. Metaheuristic-based deep COVID-19 screening model from chest X-ray images. J. Healthc. Eng. 2021, 8829829 (2021).

53. Singh, D., Kumar, V. \& Kaur, M. Densely connected convolutional networks-based COVID-19 screening model. Appl. Intell. 51, 3044-3051 (2021). 
54. Gianchandani, N., Jaiswal, A., Singh, D., Kumar, V. \& Kaur, M. Rapid COVID-19 diagnosis using ensemble deep transfer learning models from chest radiographic images. J. Ambient Intell. Human. Comput. https://doi.org/10.1007/s12652-020-02669-6 (2020).

\section{Author contributions}

Z.Z. initialized, conceived and supervised the project. Z.Z. and B.C. developed the method. B.C. extracted and preprocessed the data, conducted the experiment, analyzed the results and drafted the manuscript. J.S. and Y.L. assisted the entire experiment. Z.Z., J.S. and Y.L. provided constructive suggestions to the manuscript. All the authors provided critical review of the manuscript and approved the final draft for publication.

\section{Funding}

This work was supported in part by the National Natural Science Foundation under Grant 61976096, Guangdong Basic and Applied Basic Research Foundation under Grant 2020B1515120047, the Guangdong Foundation for Distinguished Young Scholars under Grant 2017A030306009, the Guangdong Special Support Program under Grant 2017TQ04X475, SCUT-Tianxiagu Joint Lab Funding under Grant x2zdD8212590, the Scientific Research Starting Foundation of South China University of Technology, the National Key Research and Development Program of China under Grant 2017YFB1002505, Guangdong Key Research and Development Program under Grant 2018B030339001, Guangdong Natural Science Foundation Research Team Program 1414060000024.

\section{Competing interests}

The authors declare no competing interests.

\section{Additional information}

Correspondence and requests for materials should be addressed to Z.Z.

Reprints and permissions information is available at www.nature.com/reprints.

Publisher's note Springer Nature remains neutral with regard to jurisdictional claims in published maps and institutional affiliations.

(c) Open Access This article is licensed under a Creative Commons Attribution 4.0 International License, which permits use, sharing, adaptation, distribution and reproduction in any medium or format, as long as you give appropriate credit to the original author(s) and the source, provide a link to the Creative Commons licence, and indicate if changes were made. The images or other third party material in this article are included in the article's Creative Commons licence, unless indicated otherwise in a credit line to the material. If material is not included in the article's Creative Commons licence and your intended use is not permitted by statutory regulation or exceeds the permitted use, you will need to obtain permission directly from the copyright holder. To view a copy of this licence, visit http://creativecommons.org/licenses/by/4.0/.

(C) The Author(s) 2021 